\title{
Propagation of Singularities for Semilinear Weakly Hyperbolic Differential Equations
}

\author{
Michael Dreher \\ Institute of Mathematics, University of Tsukuba \\ Tsukuba-shi, Ibaraki 305-8571, Japan \\ email: dreher@math.tsukuba.ac.jp
}

\begin{abstract}
We compare the singularities for solutions to linear and semilinear weakly hyperbolic equations with sharp Levi conditions. ${ }^{1}$
\end{abstract}

\section{Introduction}

It is known that the strongest singularities of a solution to a semilinear wave equation coincide with the singularities of a solution to the corresponding linear wave equation. This means the following. Consider the Cauchy problems

$$
\begin{aligned}
& \square u:=\left(\partial_{t t}^{2}-\triangle_{x}\right) u=f(u), \quad u(x, 0)=\varphi(x), \quad u_{t}(x, 0)=\psi(x), \\
& \square v=0, \quad v(x, 0)=\varphi(x), \quad v_{t}(x, 0)=\psi(x),
\end{aligned}
$$

where $(x, t) \in \mathbb{R}^{n+1}$ and $f \in C^{\infty}$ with $f(0)=0$. Assume that $\varphi \in H^{s}\left(\mathbb{R}^{n}\right)$, $\psi \in H^{s-1}\left(\mathbb{R}^{n}\right)$ with $s>n / 2+1$. Then it is well-known that unique solutions exist,

$$
v, u \in C\left((-T, T), H^{s}\right) \cap C^{1}\left((-T, T), H^{s-1}\right), \quad T>0 .
$$

Suppose that the initial data $\varphi(x), \psi(x)$ are smooth for $|x|>R$. Clearly, the singularities for $|x| \leq R$ propagate with speed 1. If two singularities, starting from two different points in the initial plane, meet at some (positive) time,

\footnotetext{
${ }^{1}$ AMS Classification: 35L15, 35L70, 35L80. Keywords: Singularities, semilinear weakly hyperbolic problems, loss of regularity
} 
they ignore each other in the linear case and continue their track. This implies that the function $v(x, t)$ is smooth in the lacuna $\mathcal{L}_{R}=\{(x, t):|x|<|t|-R\}$. However, in the semilinear case, the nonlinearity induces the two singularities to interact and new singularities in $\mathcal{L}_{R}$ are generated. But these new singularities are weaker, which can be seen as follows. We have

$$
\square(u-v)=f(u) \in C\left((-T, T), H^{s}\right), \quad(u-v)(0)=(u-v)_{t}(0)=0,
$$

which implies $u-v \in C\left((-T, T), H^{s+1}\right)$. Hence the singularities of $u$ in the lacuna $\mathcal{L}_{R}$ are at least one Sobolev order weaker than the singularities of $v$. (Actually, a more detailed microlocal analysis shows that the difference of smoothness is about $s-n / 2$ Sobolev orders [Rau79].)

Our goal is to show that the same comparison between solutions of linear and semilinear equations holds also in the weakly hyperbolic case.

Let us recall the definition of weak and strict hyperbolicity: An Operator $P=\partial_{t t}^{2}-\sum_{i, j=1}^{n} a_{i j}(x, t) \partial_{x_{i}} \partial_{x_{j}}$ is called weakly hyperbolic if

$$
\sum_{i, j=1}^{n} a_{i j}(x, t) \xi_{i} \xi_{j} \geq 0 \quad \forall(x, t, \xi) \in \mathbb{R}^{n} \times \mathbb{R} \times \mathbb{R}^{n} .
$$

The operator $P$ is strictly hyperbolic if

$$
\sum_{i, j=1}^{n} a_{i j}(x, t) \xi_{i} \xi_{j} \geq \alpha|\xi|^{2} \quad \forall(x, t, \xi) \in \mathbb{R}^{n} \times \mathbb{R} \times \mathbb{R}^{n}, \quad \alpha>0
$$

Singularities of solutions to weakly hyperbolic equations are particularly interesting for the following reason. In [TT80], Taniguchi and Tozaki studied equations of the form

$$
v_{t t}-t^{2 l} v_{x x}-b t^{l-1} v_{x}=0, \quad x \in \mathbb{R}^{1}, \quad l \in \mathbb{N}_{+} .
$$

Among other results, they proved that the propagation of singularities depends on the value of the real constant $b$ in a very sensitive way. Assume that the data at $t=0$ are singular at one isolated point. In general, this singularity propagates along both characteristics that start at this point. But for certain discrete values of $b$ and for certain initial data, the singularity propagates along one characteristic only.

On the other hand, the propagation of singularities in the semilinear case

$$
u_{t t}-t^{2 l} u_{x x}-b t^{l-1} u_{x}=f(u)
$$

seems to be a completely open problem. We are not aware of any results in this direction. 
In this paper, we will answer this open question for (1.2) and for much more general equations. Roughly speaking, we can show that the strongest singularities of $u$ coincide with the singularities of $v$.

The reader might argue that the choice of coefficients in (1.1) seems to be a bit artificial. However, our choice of exponents reflects the so-called Levi conditions. These are algebraical conditions between principal part and lower order terms and are necessary and sufficient for $C^{\infty}$ well-posedness, see [IP74] or [Miz85]. If, e.g., the exponent of the coefficient of $v_{x}$ is less than $l-1$, then one can construct $C^{\infty}$ data $\varphi, \psi$ with the property that no solution $v$ from $C^{2}\left(\mathbb{R}, C^{\infty}(\mathbb{R})\right)$ exists.

In order to describe the main obstacle in the investigations of (1.2), we study (1.1) more closely. As it is shown in [TT80], one can construct the solution $v$ explicitly. To do so, we apply partial Fourier transform with respect to $x$ to (1.1) and get a family of ODEs with parameter $\xi$. After several changes of variables one obtains a confluent hypergeometric differential equation. Thus, it can be shown that

$$
\begin{aligned}
\hat{v}(\xi, t)= & e^{-i \Lambda(t) \xi}{ }_{1} \mathrm{~F}_{1}\left(\frac{l+b}{2(l+1)}, \frac{l}{l+1}, 2 i \Lambda(t) \xi\right) \hat{\varphi}(\xi) \\
& +t e^{-i \Lambda(t) \xi}{ }_{1} \mathrm{~F}_{1}\left(\frac{l+2+b}{2(l+1)}, \frac{l+2}{l+1}, 2 i \Lambda(t) \xi\right) \hat{\psi}(\xi),
\end{aligned}
$$

where $\Lambda(t):=\int_{0}^{t} \tau^{l} d \tau=t^{l+1} /(l+1)$. The confluent hypergeometric function ${ }_{1} \mathrm{~F}_{1}(\alpha, \beta, z)$ behaves asymptotically like

$$
{ }_{1} \mathrm{~F}_{1}(\alpha, \beta, z) \sim \frac{\Gamma(\beta)}{\Gamma(\beta-\alpha)} e^{ \pm i \pi \alpha} z^{-\alpha}+\frac{\Gamma(\beta)}{\Gamma(\alpha)} e^{z} z^{\alpha-\beta}, \quad|z| \rightarrow \infty
$$

This can be interpreted in the following way. The factor $\exp (z)$ in the second item on the right distinguishes between the two characteristics. If $\beta-\alpha$ (or $\alpha$ ) is a negative integer, then the first (or the second) item on the right of (1.4) vanishes. Consequently, there are situations in which singularities propagate along one characteristic only. But, (1.4) contains also factors $\xi^{-\alpha}$, $\xi^{\alpha-\beta}$. Generally, one of the exponents $-\alpha, \alpha-\beta$ will be positive. In other words, a pseudodifferential operator of positive order is applied to $\varphi$ and $\psi$ in (1.3), leading to a reduced smoothness of $v$ in comparison with $\varphi, \psi$.

This so-called loss of regularity is a severe difficulty for studying semilinear equations. To explain this observation more clearly, we consider a special case. Let us fix $l=1, b=4 m+1, m \in \mathbb{N}_{0}$ and $\psi \equiv 0$. Then we have

$$
v_{t t}-t^{2} v_{x x}-(4 m+1) v_{x}=0, \quad v(x, 0)=\varphi(x), \quad v_{t}(x, 0)=0 .
$$


Qi Min-You [Qi58] constructed the solution explicitly:

$$
v(x, t)=\sum_{j=0}^{m} C_{j m} t^{2 j} \partial_{x}^{j} \varphi\left(x+t^{2} / 2\right), \quad C_{m m} \neq 0 .
$$

We observe the following:

- Singularities propagate along the characteristic $x+t^{2} / 2=$ const only.

- The solution has lost $m$ derivatives. If $\varphi \in H^{s}$, then $v(., t) \in H^{s-m}$. There is no classical solution for $m>s-5 / 2$ !

Let us have a look at the corresponding semilinear problem:

$$
u_{t t}-t^{2} u_{x x}-(4 m+1) u_{x}=f(u), \quad u(x, 0)=\varphi(x), \quad u_{t}(x, 0)=0 .
$$

It is clear that the usual iteration approach cannot work: consider a sequence $\left(u^{k}\right)$ with $u^{0}(x, t)=\varphi(x)$ and

$$
u_{t t}^{k}-t^{2} u_{x x}^{k}-(4 m+1) u_{x}^{k}=f\left(u^{k-1}\right), \quad u^{k}(x, 0)=\varphi(x), \quad u_{t}^{k}(x, 0)=0 .
$$

We see that $u^{k}(., t) \in H^{s-m k}$. In other words, even the mere existence of a solution $u$ is not clear.

There are two approaches to overcome this problem. The first approach is based on the observation that the loss of regularity does not happen if the Cauchy problem has a very special structure: the initial data must vanish, and the right-hand side must go to zero sufficiently fast, as $t$ approaches zero. It is possible to transform (1.2) into such a special Cauchy problem. During this transformation procedure, a lot of derivatives of the solution are lost. Finally, the usual iteration scheme can be applied to the new semilinear problem. This idea has been widely used in the past, compare [Ner66], [Ole70], [KY98], [DR98], [DDF98]. However, this approach gives only rough information on the smoothness of the solution $u$. Since we want to describe the smoothness and singularities of $u$ precisely, we have to find other ideas. The second approach seems to be quite new, the only reference we know is [RY97]. Its main idea is to construct a Banach space $B$ that absorbs the loss of regularity. This means that the mapping $u^{k-1} \mapsto u^{k}$ from (1.7) maps $B$ continuously into itself. The elements of $B$ have $H^{s}$ smoothness with respect to $x$ for $t=0$ and $H^{s-m}$ smoothness for every $t>0$. This space $B$ consists of functions $w(x, t)$ for those

$$
\vartheta(\xi, t) \hat{w}(\xi, t) \in C\left([0, T], L^{2}\left(\mathbb{R}_{\xi}^{n}\right)\right)
$$


holds, where $\vartheta=\vartheta(\xi, t)$ is a continuous temperate weight function with

$$
\vartheta(\xi, t)=\left\{\begin{array}{lll}
\mathcal{O}\left(\langle\xi\rangle^{s}\right) & : & t=0, \\
\mathcal{O}\left(\langle\xi\rangle^{s-m}\right) & : & t>0,
\end{array} \quad|\xi| \rightarrow \infty .\right.
$$

Let us formulate the consequences of the main result (Theorem 1) in the special cases of (1.5), (1.6):

Proposition 1 Let $f=f(u)$ be an entire analytic function. Suppose $\varphi \in$ $H^{s}\left(\mathbb{R}^{1}\right)$ with a sufficiently large $s$. Then there is a $T>0$ with the property that solutions $v, u$ to (1.5) and (1.6) exist and

$$
u, v \in C\left([0, T], H^{s-m}\right), \quad u-v \in C\left([0, T], H^{s-m+1 / 2}\right) .
$$

\section{The main result}

Now we devote ourselves to the general case. We want to compare smoothness and singularities of the solutions to the Cauchy problems

$$
\begin{aligned}
L v= & 0, \quad v(x, 0)=\varphi(x), \quad v_{t}(x, 0)=\psi(x), \\
L u= & f(u), \quad u(x, 0)=\varphi(x), \quad u_{t}(x, 0)=\psi(x), \\
L:= & \partial_{t t}^{2}-2 \sum_{j=1}^{n} \lambda(t) c_{j}(t) \partial_{x_{j} t}^{2}+\sum_{i, j=1}^{n} \lambda(t)^{2} a_{i j}(t) \partial_{x_{i} x_{j}}^{2} \\
& +\sum_{j=1}^{n} \lambda^{\prime}(t) b_{j}(t) \partial_{x_{j}}+c_{0}(t) \partial_{t} .
\end{aligned}
$$

The function $\lambda=\lambda(t)$ describes the degeneracy and has a zero of finite or infinite order at $t=0$. Let us list the assumptions:

$$
\begin{aligned}
& \lambda(t), c_{j}(t), a_{i j}(t), b_{j}(t), c_{0}(t) \in C^{\infty}([0, T]), \\
& \lambda(0)=0, \quad \lambda^{\prime}(t)>0, \quad 0<t \leq T, \\
& d_{0} \frac{\lambda(t)}{\Lambda(t)} \leq \frac{\lambda^{\prime}(t)}{\lambda(t)} \leq d_{1} \frac{\lambda(t)}{\Lambda(t)}, \quad 0<t \leq T, \quad d_{0} \geq \frac{1}{2}, \quad \Lambda(t):=\int_{0}^{t} \lambda(\tau) d \tau, \\
& \left|\partial_{t}^{k} \lambda(t)\right| \leq d_{k}\left(\frac{\lambda(t)}{\Lambda(t)}\right)^{k}, \quad 0<t \leq T, \quad k=2,3, \ldots, \\
& \left(\sum_{j=1}^{n} c_{j}(t) \xi_{j}\right)^{2}+\sum_{i, j=1}^{n} a_{i j}(t) \xi_{i} \xi_{j} \geq \alpha|\xi|^{2}, \quad \alpha>0, \quad \forall(\xi, t),
\end{aligned}
$$




$$
f(u)=\sum_{j=1}^{\infty} f_{j} u^{j} \quad \forall u \in \mathbb{R} .
$$

Examples for the weight function $\lambda=\lambda(t)$ are

$$
\lambda(t)=t^{l}, \quad l \in \mathbb{N}_{+}, \quad \lambda(t)=\exp \left(-\exp \ldots \exp |t|^{-r}\right), \quad r \in \mathbb{R}_{+} .
$$

Special linear cases with $\lambda(t)=t^{l}$ and $\lambda(t)=\partial_{t} \exp \left(-|t|^{-1}\right)$ have been studied by Taniguchi and Tozaki [TT80] and Aleksandrian [Ale84].

Before we can formulate the main result, we need some preparations.

We have to fix the temperate weight function $\vartheta(\xi, t)$ mentioned in the introduction. This weight function shall have different asymptotic behaviours for $|\xi| \rightarrow \infty$ in the two cases $t=0$ and $t>0$. Therefore we split the $(\xi, t)$-space into two zones, the so-called hyperbolic zone and the pseudodifferential zone:

$$
\begin{aligned}
& Z_{\text {hyp }}:=\{(\xi, t):|\xi| \geq 1, \quad \Lambda(t)\langle\xi\rangle \geq N\}, \\
& Z_{p d}:=\{(\xi, t):|\xi| \geq 1, \quad \Lambda(t)\langle\xi\rangle \leq N\}, \quad\langle\xi\rangle:=\left(1+|\xi|^{2}\right)^{1 / 2} .
\end{aligned}
$$

Here, $N \gg 1$ is some suitably chosen number. The border between the two zones consists of points $\left(\xi, t_{\xi}\right)$, where $t_{\xi}$ is defined by $\Lambda\left(t_{\xi}\right)\langle\xi\rangle:=N$. In the examples of [TT80] and [Ale84], $\lambda(t)=t^{l}$ and $\lambda(t)=\partial_{t} \exp \left(-|t|^{-1}\right)$, respectively, we have $t_{\xi}=\mathcal{O}\left(\langle\xi\rangle^{-1 /(l+1)}\right), t_{\xi}=\mathcal{O}(1 / \ln \langle\xi\rangle)$, respectively. We will define the weight $\vartheta(\xi, t)$ in the two zones differently in order to describe the loss of regularity.

In $Z_{p d}$, we define the auxiliary function

$$
\varrho(\xi, t):=\sqrt{1+\langle\xi\rangle \lambda(t)^{2} / \Lambda(t)}
$$

(The conditions on $\lambda$ imply that $\varrho$ is continuous for $t \rightarrow 0$.) And for the considerations in $Z_{\text {hyp }}$, we introduce the function

$$
J(s, t):=\exp \left(\int_{s}^{t} \sup _{\zeta, \pm} \frac{\lambda^{\prime}(\tau)}{2 \lambda(\tau)}\left|1 \pm \frac{b(\zeta, \tau)+c(\zeta, \tau)}{\sqrt{c(\zeta, \tau)^{2}+a(\zeta, \tau)}}\right| d \tau\right)
$$

where

$$
\begin{aligned}
b(\xi, t) & :=-\sum_{j=1}^{n} b_{j}(t) \frac{\xi_{j}}{|\xi|}, \quad c(\xi, t):=\sum_{j=1}^{n} c_{j}(t) \frac{\xi_{j}}{|\xi|}, \\
a(\xi, t) & :=\sum_{i, j=1}^{n} a_{i j}(t) \frac{\xi_{i} \xi_{j}}{|\xi|^{2}} .
\end{aligned}
$$


Now we are in a position to fix the weight function $\vartheta(\xi, t)$. In depends on nonnegative real parameters $M, K_{1}, K_{2}$ :

$$
\vartheta_{M K_{1} K_{2}}(\xi, t)= \begin{cases}\frac{\varrho\left(\xi, t_{\xi}\right)}{\varrho(\xi, t)} J\left(t_{\xi}, t_{0}\right)\langle\xi\rangle^{M} t_{\xi}^{K_{1}} & :(\xi, t) \in Z_{p d}, \\ J\left(t, t_{0}\right)\langle\xi\rangle^{M} t_{\xi}^{K_{2}} & :(\xi, t) \in Z_{\text {hyp }} .\end{cases}
$$

Here, $t_{0}$ is given by $\Lambda\left(t_{0}\right)\langle 0\rangle=N$. Then we can set

$$
\begin{aligned}
B_{M K_{1} K_{2}}:= & \left\{v \in C\left([0, T], \mathcal{S}^{\prime}\left(\mathbb{R}^{n}\right)\right): \vartheta_{M K_{1} K_{2}} \hat{v} \in C\left([0, T], L^{2}\left(\mathbb{R}_{\xi}^{n}\right)\right)\right\} \\
\|v\|_{B_{M K_{1} K_{2}}} & :=\sup _{[0, T]}\left\|\vartheta_{M K_{1} K_{2}}(., t) \hat{v}(., t)\right\|_{L^{2}\left(\mathbb{R}_{\xi}^{n}\right)} .
\end{aligned}
$$

Finally, we introduce a pseudodifferential operator $H\left(D_{x}, t\right)$ with symbol

$$
\begin{aligned}
& h(\xi, t):=\lambda(t)|\xi| \chi(\Lambda(t)|\xi| / N)+\varrho(\xi, t)(1-\chi(\Lambda(t)|\xi| / N)), \\
& \chi(s)=0 \quad(s \leq 1), \quad \chi(s)=1 \quad(s \geq 2), \quad \chi \in C^{\infty}(\mathbb{R}) .
\end{aligned}
$$

For fixed $t>0, H\left(D_{x}, t\right)$ behaves like $\lambda(t)\left\langle D_{x}\right\rangle$.

Now we have all notations for the main result.

Theorem 1 Suppose that $M, N$ are sufficiently large and assume that $H\left(D_{x}, 0\right) \varphi \in B_{M K K}$ and $H\left(D_{x}, 0\right) \psi \in B_{M(K+1) K}$. If $T>0$ is small enough, then there exist solutions $v, u$ to (2.1), (2.2) and

$$
\begin{aligned}
& H\left(D_{x}, t\right) u, \quad H\left(D_{x}, t\right) v \in B_{M K K}, \quad D_{t} u, \quad D_{t} v \in B_{M(K+1) K}, \\
& H\left(D_{x}, t\right)(u-v) \in B_{M(K-1)(K-1)}, \quad D_{t}(u-v) \in B_{M(K-1)(K-1)} .
\end{aligned}
$$

Remark 1 In the cases in which an explicit representation of $v$ is known (compare [TT80] and [Ale84]), this theorem yields exactly the same loss of regularity as predicted by the explicit representation.

Remark 2 The regularity of $u-v$ is higher than that of $u, v$. The difference of regularity is given by the pseudodifferential operator with symbol $t_{\xi}$.

\section{Sketch of proof}

For details, we refer the reader to $[\mathrm{DR}]$. We start from the Cauchy problem

$$
L w=g(x, t), \quad w(x, 0)=\varphi(x), \quad w_{t}(x, 0)=\psi(x) .
$$


Employing partial Fourier transform with respect to $x$ gives us an ODE with parameter $\xi$. We study this ODE in the two zones separately. In $Z_{p d}$, we transform the ODE into a first order system for the vector of unknowns

$$
W(\xi, t)=\left(\varrho(\xi, t) \hat{w}(\xi, t), D_{t} \hat{w}(\xi, t)\right)^{T} .
$$

A quite technical investigation of this system reveals that

$$
\begin{gathered}
|\varrho(\xi, t) \hat{w}(\xi, t)| \leq C \varrho(\xi, t)\left(\int_{0}^{t}(t-s)|\hat{g}(\xi, s)| d s+|\hat{\varphi}(\xi)|+t|\hat{\psi}(\xi)|\right), \\
\left|D_{t} \hat{w}(\xi, t)\right| \leq C \int_{0}^{t}(1+(t-s)(\lambda(t)-\lambda(s))\langle\xi\rangle)|\hat{g}(\xi, s)| d s \\
+C \lambda(t)\langle\xi\rangle|\hat{\varphi}(\xi)|+C(1+t \lambda(t)\langle\xi\rangle)|\hat{\psi}(\xi)| .
\end{gathered}
$$

In $Z_{\text {hyp }}$, we obtain a first order system for the vector

$$
W(\xi, t)=\left(\lambda(t)|\xi| \hat{w}(\xi, t), D_{t} \hat{w}(\xi, t)\right)^{T} .
$$

Using ideas from [Yag97], we perform a diagonalization process and obtain

$$
\begin{aligned}
& |\lambda(t) \xi \hat{w}(\xi, t)|+\left|D_{t} \hat{w}(\xi, t)\right| \\
& \quad \leq C \int_{t_{\xi}}^{t} J(s, t)|g(\hat{\xi}, s)| d s+C J\left(t_{\xi}, t\right)\left(\left|\lambda\left(t_{\xi}\right) \xi \hat{w}\left(\xi, t_{\xi}\right)+\right| D_{t} \hat{w}\left(\xi, t_{\xi}\right) \mid\right) .
\end{aligned}
$$

Exploiting these pointwise estimates, one can show

$$
\begin{aligned}
& \left\|H\left(D_{x}, t\right) w\right\|_{B_{M K K}}+\left\|D_{t} w\right\|_{B_{M(K+1) K}} \\
& \quad \leq C\left(T\|g\|_{B_{M K K}}+\left\|H\left(D_{x}, 0\right) \varphi\right\|_{B_{M K K}}+\left\|H\left(D_{x}, 0\right) \psi\right\|_{B_{M(K+1) K}}\right) .
\end{aligned}
$$

This proves the statements for $v$.

We observe that the norms of $H w$ and $g$ in that estimate are the same. This means that our choice of function spaces has absorbed the loss of regularity. One can show that $B_{M K K}$ is a multiplicative algebra if $M$ is large. Then the function $u \mapsto f(u)$ maps $B_{M K K}$ into itself. Consequently, a solution $u$ to (2.2) exists for small $T>0$ and has the claimed smoothness.

It remains to study $u-v$. Obviously, $u-v$ solves $L(u-v)=f(u)$ with vanishing initial data. The function $u$ belongs to a slightly better space than $H u$; namely the space for $u$ is described by the temperate weight $\vartheta_{M K K}(\xi, t) / h(\xi, t)$. This space for $u$ is also a multiplicative algebra. By proving a similar a-priori-estimate to that for $w$, it is possible to show that $u-v$ has higher regularity than $u$ and $v$.

Acknowledgement The author would like to thank Prof. Kajitani from Tsukuba University for many discussions on the subject of this paper. 


\section{References}

[Ale84] G. Aleksandrian. Parametrix and propagation of the wave front of a solution to a Cauchy problem for a model hyperbolic equation (in Russian). Izv. Akad. Nauk Arm. SSR, 19(3):219-232, 1984.

[DDF98] P. D'Ancona and M. Di Flaviano. On quasilinear hyperbolic equations with degenerate principal part. Tsukuba J. Math., 22(3):559$574,1998$.

[DR] M. Dreher and M. Reissig. Propagation of singularities for semilinear weakly hyperbolic differential equations. to appear.

[DR98] M. Dreher and M. Reissig. Local solutions of fully nonlinear weakly hyperbolic differential equations in Sobolev spaces. Hokk. Math. J., 27(2):337-381, 1998.

[IP74] Ivrii, V.Ya. and Petkov, V.M. Necessary conditions for the Cauchy problem for non-strictly hyperbolic equations to be well-posed (in Russian). Uspekhi Mat. Nauk, 29(5):3-70, 1974. English translation: Russian Math. Surveys 29(5):1-70, 1974.

[KY98] K. Kajitani and K. Yagdjian. Quasilinear hyperbolic operators with the characteristics of variable multiplicity. Tsukuba J. Math., 22(1):49-85, 1998.

[Miz85] S. Mizohata. On the Cauchy Problem. Science Press, Academic Press, 1985.

[Ner66] A. Nersesyan. On a Cauchy problem for degenerate hyperbolic equations of second order (in Russian). Dokl. Akad. Nauk SSSR, 166(6):1288-1291, 1966.

[Ole70] O.A. Oleinik. On the Cauchy problem for weakly hyperbolic equations. CPAM, 23:569-586, 1970.

[Qi58] Qi Min-You. On the Cauchy problem for a class of hyperbolic equations with initial data on the parabolic degenerating line. Acta Math. Sinica, 8:521-529, 1958.

[Rau79] J. Rauch. Singularities of solutions to semilinear wave equations. J. Math. Pures Appl., 58:299-308, 1979. 
[RY97] M. Reissig and K. Yagdjian. Weakly hyperbolic equations with fast oscillating coefficients. Preprint 7, Technische Universität Bergakademie Freiberg, 1997.

[TT80] K. Taniguchi and Y. Tozaki. A hyperbolic equation with double characteristics which has a solution with branching singularities. Math. Japonica, 25(3):279-300, 1980.

[Yag97] K. Yagdjian. The Cauchy Problem for Hyperbolic Operators. Multiple Characteristics, Micro-Local Approach. Akademie Verlag, Berlin, 1997. 